\title{
Factores determinantes de la calidad del cuidado asistencial institucional a personas mayores y/o con discapacidad
}

\author{
Julián Montoro Rodríguez \\ Maned School of Applied Social Science. Care Western Reserve University \\ 10900 Euclid Aveuve. Cleveland. Ohio (USA) 44106 - 7164 \\ julian@offcampus.es \\ JRM11@po-cwru.edu
}

\section{Resumen}

Los estudios de investigación sociológica de ambos enfoques, macrosocial y microsocial, han señalado diversos factores que determinan el resultado o la calidad del cuidado institucional en las residencias para ancianos. El presente estudio propone un modelo inicial sobre el cuidado institucional y analiza la influencia de los factores predictivos en la calidad asistencial sobre una muestra de cien residencias en EE.UU. que incluye 286 residentes y sus familiares. Los factores más importantes asociados con la calidad asistencial en el presente estudio fueron los aspectos del nivel institucional - el carácter privado del centro, el medio ambiente, la configuración familiar, el número de profesionales, su formación y entrenamiento para combatir el estrés laboral y grado de retención laboral—, seguidos de los indicadores del nivel relacional — participación y cuidado informal de los familiares - Sin embargo, en contra de lo que se esperaba, los factores del nivel interaccional — habilidad de comunicación y conflicto personal-, no fueron significativos respecto de la calidad asistencial, o al menos no parecen tener una influencia decisiva según los resultados analizados.

Palabras clave: calidad asistencial, residencias, ancianos, cuidado institucional.

\begin{abstract}
Sociological studies on both the macro and the micro level have pointed out different factors that affect the quality of institutional care provided to elderly in Nursing Homes. This study proposes a theoretical basic model of quality of care, and examines the influence of predictive factors on the quality of assisting on a sample of 100 Nursing Homes in the USA, including 286 residents and their family caregivers. The most important factors associated with the quality of care in this study were several aspects of the institutional level —whether the center is for profit, the environment, familiar configuration, percentage of staff per bed, stress training, and turnover-, followed by relational factors-family involvement and participation. However, contrary to expectations, factors of the interactional level —communication styles, personal conflict resolution-, were not significant on the quality of institutional care, or at least, based on the results of this study, they did not seem to have a significant influence on the level of quality of care.
\end{abstract}

Key words: Quality of Care, Nursing Homes, Elderly, Institutional Care. 


\section{Sumario}

\begin{tabular}{|c|c|}
\hline \multirow{2}{*}{$\begin{array}{r}\text { Introducción } \\
\text { Sociología y cuidado institucional } \\
\text { a personas mayores }\end{array}$} & $\begin{array}{l}\text { Modelo teórico del cuidado asistencial } \\
\text { institucional }\end{array}$ \\
\hline & Estudio: material y método \\
\hline $\begin{array}{r}\text { Factores que determinan la calidad del } \\
\text { cuidado institucional }\end{array}$ & Resultados \\
\hline Impactos de la asistencia institucional & Discusión \\
\hline a los mayores & Bibliografía \\
\hline
\end{tabular}

\section{Introducción}

Según el Manual estadístico sobre el envejecimiento de la población en España editado por la Fundación Caja Madrid en 1996, casi un 3\% de los mayores residen en centros o instituciones para la tercera edad. Este tipo de alojamiento colectivo se convierte en un recurso para las personas mayores que están en situación de necesidad, de dependencia, y que no poseen un apoyo familiar o informal al que recurrir. La residencia representa un centro de atención sociosanitario integral que sirve a un determinado perfil de personas mayores, en particular a aquéllas que no son capaces de valerse por sí mismas o no pueden ser atendidas en sus domicilios.

Los residentes de este tipo de centros sociosanitarios declaran en general sentirse satisfechos del servicio que reciben en las residencias, sin embargo, ciertos estudios cualitativos sobre la calidad de vida en las mismas nos indican que existen aspectos negativos en el cuidado institucional. La imagen que surge sugiere que este tipo de atención no es el más deseado entre los mayores. De igual modo se expresan los mismos residentes con respecto a la atención institucional: "para los residentes las residencias son la solución menos mala, son vistas como un mal menor» (Bazo, 1991). Parece pues que las residencias para la tercera edad no son consideradas como una solución muy atractiva por los españoles. Según el estudio número 1.792 del Centro de Investigaciones Sociológicas (CIS), basado en una muestra de la población española en 1989, tan sólo el 10\% querría vivir en una residencia, y ocho de cada diez encuestados, que en la actualidad no viven en residencias, indican que aunque pudieran, no desearían residir en ellas.

Una actitud muy similar se desprende igualmente de los resultados de la encuesta a la población de 65 y más años del CIS (INSERSO, 1995). A pesar de que un $79 \%$ dice conocer este tipo de servicio público, algo más de dos tercios de los que dicen conocer las residencias afirman: «que no les interesan, no les gustan», y otro adicional $7 \%$ expresa de forma directa su rechazo a las mismas afirmando que: «no son un lugar para mí». Las residencias reciben igualmente la valoración más baja de entre los servicios públicos de asistencia, tan sólo el 63\% valoran moderadamente («bien») su funcionamiento frente a un $90 \%$ que otorga similar valoración a los servicios de vacaciones, balnearios, hogares y clubes de ancianos. 
Lo que se desprende de estos datos es una imagen negativa de las residencias en cuanto hogares colectivos entre los adultos. La cuestión entonces es preguntarse por las causas que han provocado esta imagen, así como las condiciones sobre las que se puede intervenir para modificar la actitud de rechazo o consideración de este servicio como último recurso para los mayores necesitados.

En España, recientemente se ha realizado un crecimiento importante en el número de plazas de residencia, que ha pasado de 106.485 en 1988 a 163.338 en 1994 , con un crecimiento en la cobertura de 2,1 plazas por cada cien mayores en 1988 a 2,8 en 1994. Estos niveles de porcentajes están por debajo de las recomendaciones realizadas por el Plan Gerontológico de 1992, así como de la media de los países europeos, que se sitúa entre 5 y 6 plazas por cada cien mayores. La tendencia pues en la oferta de residencias y su cobertura por cada cien mayores es de esperar que sea la de un incremento de dichas plazas, en particular de aquéllas que corresponden a la iniciativa privada.

$\mathrm{El}$ reto que plantea el crecimiento del servicio de residencias para mayores consiste fundamentalmente en el nivel de calidad que puedan ofrecer en cuanto alojamiento institucional colectivo. Es por ello de interés, desde el punto de vista de la política social, el examinar aquellos factores que influyen o determinan el impacto que dichas residencias van a producir, tanto sobre los residentes y su calidad de vida, como sobre las expectativas de sus familiares y la sociedad en su conjunto.

\section{Sociología y cuidado institucional a personas mayores}

La atención sociosanitaria a largo plazo de los ancianos se realiza, fundamentalmente, a través de las residencias para personas mayores de 65 años. El interés de la sociología, por dichos centros residenciales de atención a personas dependientes, se da tanto a un nivel macrosocial como microsocial:

Desde un punto de vista macrosocial, el interés se centra en el estudio de la residencias en cuanto organizaciones formales de atención sociosanitaria, los sistemas de regulación, gestión y financiación de las mismas, y/o en su carácter de servicio público. Los estudios sociológicos que mejor representan este nivel de análisis son los que utilizan el enfoque teórico denominado «Economía Política de la Vejez», desarrollado fundamentalmente por Caroll L. Estes (1979) y/o Jill Quadagno (1982) en Estados Unidos, y Alan Walker (1981) en Europa.

Los temas de análisis se centran en la influencia que ejerce el sistema político, el económico y el social sobre las condiciones de los ancianos y su acceso a los servicios sociosanitarios. En particular, analizan los procedimientos y/o mecanismos por los que dichos sistemas generan y consolidan desigualdades sociales entre las personas mayores y entre éstas y las de otros grupos de edad.

Caroll Estes y sus colaboradores (1993) han identificado al menos cuatro "procesos de transformación» que han influido en la organización y provisión de servicios tales como el de la ayuda domiciliaria de salud (Home Health Care) o el de la residencia (Nursing Homes) en los Estados Unidos. Dichos 
procesos serían: a) la privatización de la industria de los servicios; b) la medicalización y proliferación de servicios médicos más que sociales; $c$ ) el cambio en la composición y las condiciones de los profesionales de los servicios y $d$ ) otros cambios estructurales y burocráticos encaminados a la eliminación de costes $\mathrm{y}$ al incremento de beneficios.

Las políticas sociales de promoción y provisión de servicios públicos han sido objeto de atención sociológica debido no sólo a su impacto sobre la vida de los ancianos, sino también a las diversas imágenes o modelos que asumen sobre la categorización social, es decir, sobre la construcción social de las realidades de la vejez y de los ancianos que implican cada una de ellas (AttiasDonfut, 1992). Así, determinadas políticas asumen diversas imágenes y representaciones de los mayores, entre las que podemos destacar al menos dos: a) la categorización del anciano como persona dependiente, no activa, perteneciente al grupo de "viejos», y b) la propuesta que pretende el reforzamiento de la responsabilidad familiar en el cuidado de los mayores como alternativa a la escasez de financiación pública de los servicios.

Desde una perspectiva microsocial, los trabajos sociológicos se centran en el estudio de los resultados o impactos del cuidado institucional sobre los residentes, los familiares o cuidadores informales de los mismos, y los profesionales del cuidado sociosanitario. En estos estudios las residencias son organizaciones que no sólo asisten sino que también controlan y/o regulan la actividad diarias de los residentes.

Dentro de este enfoque se encuentran los trabajos que utilizan el concepto de «institución total» elaborado por Erving Goffman (1961). La aplicación de este modelo ideal al estudio de las residencias o instituciones de ancianos, se centra en el análisis de las dimensiones que mejor definen las residencias como instituciones totales. Pia Barenys (1993) señala como más importantes dos de estas dimensiones: a) el grado de internamiento o frecuencia e intensidad de intercambios con el mundo exterior, y $b$ ) el grado de reglamentación o pérdida de control personal de los residentes según su condición.

Otros estudios dentro de la perspectiva microsocial corresponden a las aportaciones de Eva y Boaz Kahana (1989) y su idea central de adaptación o competencia personal, del mantenimiento de congruencia entre la persona y el medio o contexto con el que interacciona («Person-Environment Fit»). Este modelo de análisis incluye estudios sobre las interacciones entre los residentes y los profesionales, los administradores, y los familiares y amigos.

Otro de los esquemas conceptuales utilizado con frecuencia es el modelo social formulado por R.L. Kane y R.A. Kane (1978). Éste propone como objetivos prioritarios el logro de la calidad de vida de los residentes a través de la promoción de la autonomía personal y de un ambiente familiar. La atención se considera adecuada si la asistencia provoca un mantenimiento del bienestar psicológico, de la autoestima y del nivel de satisfacción general de los mismos. El énfasis se pone en los aspectos sociales, familiares, personales más que en los médicos y/o institucionales. Los mayores son considerados más como residentes que como pacientes en la institución. 
El ingreso en una residencia y la vida prolongada en la institución tienen, sin duda efectos positivos, como por ejemplo el de recibir apoyo ante las limitaciones funcionales de los residentes; sin embargo, las investigaciones también nos indican la existencia de determinados efectos negativos, que provocan que el nivel de vulnerabilidad y de riesgo de muerte se eleve de forma considerable para los residentes. Numerosas investigaciones señalan entre éstos a: los síntomas depresivos, la soledad, y/o la despersonalización (Lawton, 1980; Kahana, Kahana y Young, 1985).

\section{Factores que determinan la calidad del cuidado institucional}

Los estudios de investigación sociológica de ambos enfoques, macrosocial y microsocial, han señalado una gran diversidad de factores que determinan el resultado o la calidad del cuidado dispensado a los residentes. En un intento de sistematizar los factores que intervienen se presenta una clasificación en tres niveles, que agrupa los aspectos más importantes detectados en la literatura, tanto gerontológica como en las otras ciencias sociales y de la salud: a) el nivel institucional, b) el nivel social o relacional, y, finalmente, c) el nivel interaccional. En la figura 1 se recogen dichos niveles, así como algunos de los factores que mejor los representan. Veamos en detalle aquellas variables que se han reconocido como significativas y relevantes para determinar la calidad del cuidado institucional.
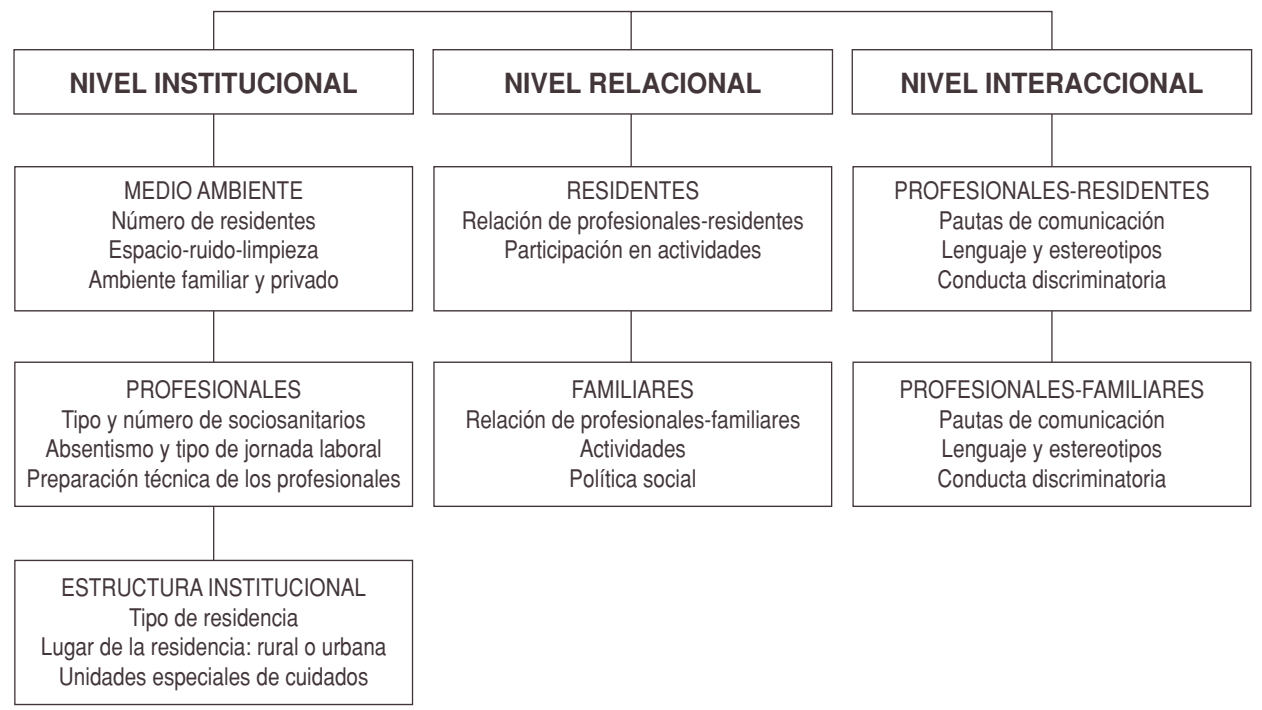

Figura 1. Factores que determinan la calidad del cuidado institucional a personas mayores dependientes. 


\section{a) Nivel institucional}

Los factores que pertenecen al ámbito institucional han sido los primeros y los más estudiados por los investigadores del área de la gerontología social. Las investigaciones más importantes examinan la influencia del entorno o medio físico de las residencias (Maslow, 1994). La idea básica es que determinados aspectos del medio ambiente son importantes para comprender la conducta y el bienestar general de los individuos. Sin embargo, a pesar de los muchos estudios realizados, todavía no existe un consenso sobre el modelo conceptual que se debe utilizar en relación con el estudio del medio, así como tampoco sobre los indicadores más significativos que se deben utilizar (George y Maddox, 1989).

Las aportaciones más importantes corresponden a tres líneas de investigación: primera, al trabajo de conceptualización y medición del medio ambiente realizado por R.H. Moos y sus colaboradores (1979; 1984), que desarrollaron la escala Multiphasic Environmental Assessment Procedure para conocer la percepción de los residentes y profesionales sobre la calidad del medio o entorno institucional; segunda, al modelo de Lawton (1989) sobre la presión del medio, que sugiere que la mayor sensibilidad a las demandas del medio provoca la aparición de enfermedades crónicas en los mayores. Lawton también desarrolló la escala Multilevel Assessment Instrument para evaluar las dimensiones del nivel funcional incluyendo el factor medioambiental; y finalmente, al modelo de congruencia entre la persona y el medio (Person-Environment Fit), elaborado por Eva y Boaz Kahana (1982), que proponen una adecuación del medio a las necesidades de los residentes.

Dentro del nivel institucional, los factores examinados para elevar la calidad del cuidado institucional se pueden agrupar en tres tipos: a) los relacionados con el diseño del medio ambiente, incluyendo variables referidas a los residentes —número, nivel de dependencia, etc.—, al espacio y entorno físico, así como su mantenimiento —iluminación, limpieza, ruidos, etc.—y, finalmente, al ambiente familiar en el medio institucional — privaticidad, objetos personales, seguridad, etc.- - $b$ ) los que se relacionan con los profesionales del centro - los gestores, personal asistencial y de servicios-, incluyendo variables tales como el tipo y el número de profesionales, el nivel de absentismo laboral, la preparación técnica, etc., y c) los que corresponden a la estructura institucional, en particular, el tipo de residencia — pública o privadasu ubicación — rural o urbana-, y/o su diferenciación interna —unidades especiales de cuidados.

\section{b) Nivel relacional}

El segundo tipo de factores se pueden considerar al nivel relacional, puesto que se analiza la relación entre el bienestar psicosocial de los residentes y la calidad de sus relaciones personales con los profesionales del centro, residentes, amigos y familiares (Noelker y Harel, 1978). 
Las variables de interés se miden con indicadores sobre la participación de los residentes y sus cuidadores familiares o informales en las actividades, programas y políticas del centro. Los administradores de las residencias pueden utilizar diversas políticas y modos de considerar a los familiares de los residentes, desde una actitud de completa ignorancia a la integración de la red social de los residentes en las decisiones y actividades del centro. Por ejemplo, el estudio de Rhonda Montgomery (1982) indica que cuando los familiares de los residentes participan en las actividades y programas del centro, los resultados son claramente positivos, dándose un mayor grado de satisfacción por parte de los familiares. Éstos son, al igual que los residentes, los verdaderos usuarios del servicio, puesto que su participación no se considera una carga sino un recurso, un beneficio para la calidad asistencial institucional.

La importancia de estos factores se basa en la evidencia de que la situación de cada residente no puede considerarse una cuestión de azar, como si su dependencia y bienestar personal actual hayan surgido de la nada, sino que cada individuo posee una trayectoria propia, cuya identidad se ha configurado con una multitud de relaciones y momentos compartidos. De sus decisiones previas, de su red social y familiar, de su formación y actividad ocupacional previos, no podemos desentendernos sin correr el riesgo de incrementar los impactos negativos de la vida en la institución.

El papel de los familiares, y de los profesionales, ha sido examinado bajo el prisma de la división del trabajo entre cuidador formal — profesional- e informal — familiar- . Este modelo, elaborado por Litwak (1985; 1990), contrapone la ayuda informal con la ayuda formal de los profesionales. Se espera que, en las residencias, los profesionales atiendan las tareas rutinarias y uniformes de asistencia, los aspectos o tareas que necesitan de un conocimiento técnico, y se deja al cuidador familiar la realización de tareas de cuidado, que no requieren de dicha técnica o preparación, el cuidado de apoyo emocional o personal.

Un enfoque alternativo es el que M.T. Duncan y D.L. Morgan (1994) nos presentan, en el sentido de que las tareas asistenciales sean compartidas y continuadas entre familiares y profesionales. En este modelo los profesionales realizan no sólo tareas de tipo técnico, sino que también asisten en los aspectos sociales y emocionales de los residentes. Los profesionales han de reconocer y aceptar el caudal de experiencia que los cuidadores familiares poseen en base al cuidado que otorgaron a su familiar antes de su institucionalización. Lo importante, desde la perspectiva de los cuidadores familiares, no es sólo el tipo de tareas que se realizan sino cómo son desempeñadas.

\section{c) Nivel interaccional}

El nivel interaccional abarca los factores que corresponden a las interacciones cara a cara que se producen entre los residentes, los profesionales del centro, los amigos y los familiares. Es el nivel básico donde se producen y se establecen relaciones constructivas o positivas (Naussbaum, 1991). Las pautas de comunicación y los estilos de resolución de conflictos son los indicadores fun- 
damentales a este nivel. La existencia de estereotipos negativos, sobre los mayores dependientes, puede influir para que éstos reciban un trato discriminatorio. El lenguaje y la conducta discriminatoria, en el cuidado institucional, son variables que se deben considerar para reforzar la calidad del cuidado institucional (Kemper, 1994).

En la medida que la institución transmita una atmósfera de colaboración y de entendimiento, no solo con los residentes sino también con sus familiares, amigos, voluntarios y demás cuidadores informales, el resultado de la acción institucional será más positivo. En general, las investigaciones indican que las pautas de comunicación entre los residentes y el personal profesional son muy negativas. Por ejemplo, el estudio de Pillemer y Hudson (1993) nos informa de que el $51 \%$ de las auxiliares de clínica y enfermería tuvieron un episodio de enfado — gritando a un residente- durante el mes anterior. Lo que se propone es que para lograr pautas de comunicación positivas son necesarias destrezas técnicas, así como habilidades para la resolución de conflictos (Pray, 1993).

Un estilo de resolución de conflictos, según nos dice Hocker y Wilmot (1995), es una pauta de respuestas, o conjunto de conductas, que los individuos utilizan en situaciones de conflicto. Se trata de una orientación comunicativa del individuo, el cual resuelve las situaciones de conflicto eligiendo las estrategias y tácticas adecuadas. Los estilos de resolución de conflicto pueden ser aprendidos por los individuos. Según Putnam y Wilson (1982), podemos reconocer al menos tres estilos de resolución de conflictos: a) actitud «controladora", que se caracteriza por una conducta agresiva y no cooperadora; b) actitud "cooperadora», que se traduce en cooperación mutua y preocupación por la relación personal, y c) la actitud de "no confrontación», que utiliza conductas de evasión o estrategias indirectas.

La idea central es que cada uno de estos estilos es apropiado para resolver diferentes situaciones; siendo la actitud cooperadora la más positiva, puesto que intenta salvar la dignidad de las dos partes. Sin embargo, la actitud de no confrontación parece ser el estilo más indicado en las residencias asistidas, debido al deterioro cognitivo y funcional de los residentes (Small, Montoro y Kemper, 1996). Por ejemplo, cuando un residente no tiene una actitud cooperadora, el personal profesional o cuidador puede actuar alejándose de una situación de conflicto o distrayendo al residente con otro tipo de actividad, y de esta manera «resuelve» la situación. Por otra parte, un estilo confrontador no sería el recomendable para la asistencia institucional a ancianos, pues conlleva una actitud negativa y puede, por lo tanto, hacer disparar la espiral de conflicto.

\section{Impactos de la asistencia institucional a los mayores}

Los factores enumerados previamente contribuyen, en diversa medida, al nivel de calidad de la asistencia institucional. Ésta depende en buena parte del cumplimiento de los objetivos generales de los centros asistenciales, entre los que hay que incluir tanto los referidos a la oferta de un espacio para los servicios básicos, como los que propician la convivencia, la integración social y el desarro- 
llo humano de los residentes. El impacto de estos factores se produce sobre la condición de los residentes, sobre los profesionales y los familiares o cuidadores informales. La calidad de la asistencia institucional es, pues, difícil de evaluar debido a su multidimensionalidad; sin embargo, podemos obtener, de forma indirecta, un índice aproximado de la misma examinando su impacto sobre los residentes, los familiares y los profesionales del centro.

Los gestores y profesionales de los centros son cada vez más conscientes de la necesidad, y del valor, de incluir a los familiares y a los cuidadores informales en las actividades y programas. Existe una gran cantidad de evidencia empírica que confirma el papel crítico que corresponde a los miembros familiares en la atención institucional. Las interacciones entre residentes, profesionales y familiares contribuyen de forma significativa en el nivel de calidad de la asistencia (Pratt y otros, 1987; Maas, Buckwalter y Kelley, 1991; Duncan y Morgan, 1994). Veamos con más detalle el tipo de resultados, o impactos, que los estudios han reconocido (en la figura 2 se muestran algunas variables más representativas).

Los estudios sobre la calidad de la asistencia institucional, que se refieren a los resultados experimentados por los residentes, examinan entre otros aspectos los siguientes: el nivel de movilidad, despersonalización, capacidad de pensamiento propio, pérdida de competencia y estima personal, dependencia, daño social, sentimientos depresivos, declive del funcionamiento psicológico y social, conducta problemática y nivel de accidentes — caidas y daños-. Los resultados negativos son más pronunciados cuando el residente tiene mayor dificultad de adaptación a las demandas de su nuevo entorno en relación con el medio familiar comunitario.

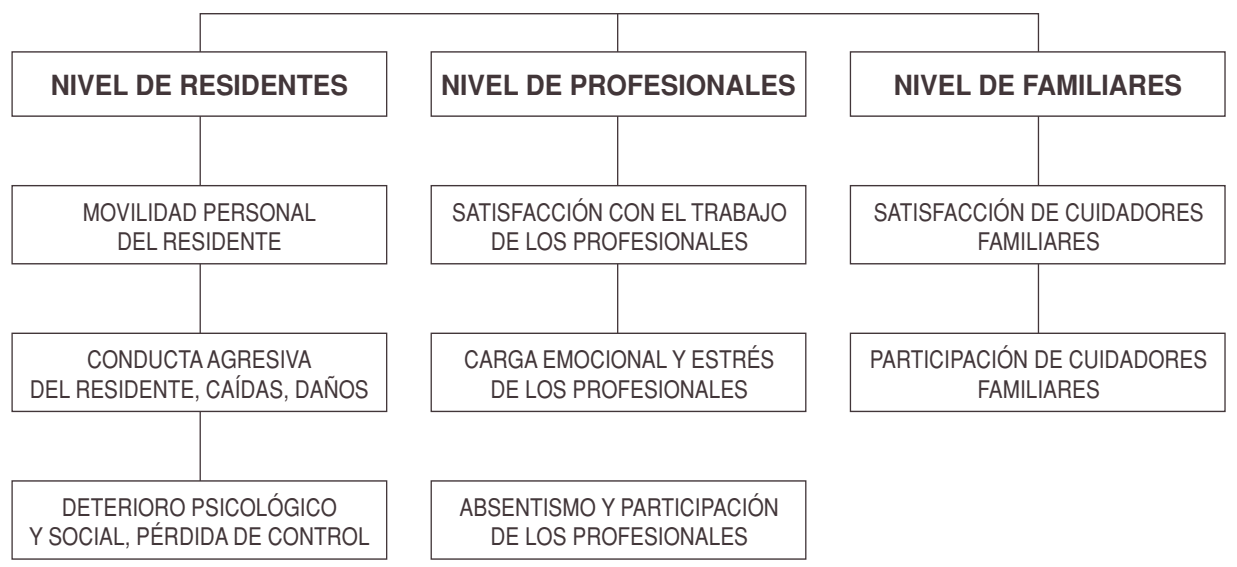

Figura 2. Impactos de la calidad del cuidado institucional a personas mayores dependientes. 
Los efectos derivados de la institucionalización en una residencia son múltiples y especialmente importantes para el residente. Dichos efectos comienzan incluso antes del ingreso en el centro (Tobin, 1989). Las personas que se disponen a ingresar en una residencia pueden experimentar un sentimiento de separación y/o un rechazo de sus familiares y amigos. El proceso de cambio de residencia, de la vivienda en la comunidad al centro institucional, conlleva una gran dosis de estrés y ansiedad. Los resultados, según las investigaciones de Lieberman y Tobin (1983), indican un alto porcentaje de mortalidad y de morbilidad entre los nuevos residentes (entre un $20 \%$ y un $30 \%$ muere durante el primer año de residencia). Los ancianos, incluso en las residencias con la más exigente calidad institucional, experimentan resultados negativos. Es por ello necesario planificar acciones formativas y estrategias, destinadas al mantenimiento psicológico y funcional de los residentes, en particular, durante los primeros meses de su institucionalización. En definitiva, tal y como Eva Kahana nos indica: "para mejorar las vidas de los residentes hay que ofrecer mejores programas nutricionales y médicos, más actividades y oportunidades de interacción social, así como también programas de estimulación cognitiva» (1987).

La calidad de la asistencia institucional tiene, también, mucho que ver con el nivel de satisfacción de los profesionales del centro — en particular con su nivel de satisfacción laboral, de carga emocional, de estrés, o grado de absentismo laboral—. Por ejemplo, según indica Itschaki (1994), el rendimiento de los trabajadores auxiliares puede verse limitado por los conflictos internos con otros trabajadores, así como por su falta de formación y entrenamiento para las tareas de asistencia que realizan (Chappell y Novak, 1992).

Otras investigaciones indican que el nivel de satisfacción de los trabajadores también está determinado por el nivel de comunicación directa entre éstos, los residentes y sus familiares (Parry y Smith, 1987; Lynch, 1994). Igualmente, los resultados preliminares de estudios sobre el impacto de las unidades especiales de cuidados (SCU) para enfermos de alzheimer, señalan como críticos a los factores relacionados con los profesionales - absentismo laboral, número de personal, etc.- - Éstos, junto al grado de satisfacción laboral, se consideran aspectos clave para una asistencia institucional de calidad (Montgomery y Montoro, 1995).

Finalmente, la calidad de la asistencia institucional tiene un impacto, igualmente importante, en los familiares y cuidadores informales de los residentes. El grado de satisfacción, el nivel de participación, el seguimiento en las actividades y programas desarrollados por el centro, nos sirven para realizar una valoración de la calidad asistencial, del impacto de la asistencia institucional (Duncan y Morgan, 1994). La percepción de los familiares, respecto de una asistencia institucional deficiente, está asociada con la constatación, por parte de los familiares, del sentimiento de ser completamente ignorados e invalidados por los profesionales del centro.

Las relaciones de los residentes y profesionales con los familiares suponen un modo de superar los aspectos más negativos de la residencia en cuanto «ins- 
titución total». Familiares y cuidadores informales son el único lazo de unión para muchos residentes con el mundo exterior. $\mathrm{Al}$ igual que se ha puesto de manifiesto la relevancia de la asociación entre la calidad de la interacción familiar y el nivel de bienestar de los mayores dependientes que residen en la comunidad (George y Maddox, 1989). Dicha asociación se supone también válida para la asistencia institucional. Muchos investigadores asumen, por lo tanto, que la interacción y participación de los familiares en la asistencia institucional está asociada positivamente con el nivel general de bienestar y del deterioro psicológico de los residentes. Sin embargo, dichas relaciones son en muchos casos conflictivas. Los profesionales y gestores de los centros pueden considerar que los familiares tienen expectativas muy poco realistas sobre el tipo y la intensidad de la asistencia que los centros pueden realmente ofrecer. En su opinión, una mayor participación de los familiares puede convertirse en un obstáculo o en una interferencia en las tareas asistenciales (Rubin y Shuttlesworth, 1983). La queja más frecuente de los familiares es la falta de preparación de los profesionales, sobre todo cuando los residentes necesitan de un cuidado especial derivado de su enfermedad —alzheimer, parkinson, etc. (Chernoweth y Spencer, 1986).

\section{Modelo teórico del cuidado asistencial institucional}

La construcción de un modelo teórico inicial sobre la asistencia institucional incluye los factores que han sido reconocidos como determinantes para la calidad del cuidado asistencial que se realiza en las residencias. La aplicación del concepto de Erving Goffman (1961), que considera la residencia como una «institución total», constituiría la base teórica del modelo, cuya hipótesis central indica que los elementos o aspectos que ayudan a hacer de la institución un centro asistencial alejado de los rasgos negativos característicos de toda «institución total», estarían asociados con una asistencia institucional con resultados positivos, tanto para los residentes como para familiares y profesionales del centro.

En la figura 3 se presentan algunas de las variables del modelo inicial que incluye las relaciones directas entre las variables independientes o factores determinantes del cuidado asistencial y las variables dependientes o resultados de la asistencia o calidad institucional. Los impactos se examinan tanto a nivel de los residentes como de familiares y profesionales.

El modelo que integra las aportaciones de los estudios examinados previamente ha de tener en cuenta igualmente ciertas variables de control, puesto que la asistencia institucional se realiza a residentes heterogéneos, diversos. Además, los residentes, como han puesto de manifiesto los estudios que utilizan el modelo de estrés y medio institucional (Kahana, Kahana y Young, 1985), no han de ser considerados como objetos receptores y pasivos, sino que más bien al contrario: son sujetos, agentes activos, que saben adaptarse a la institución otorgando un sentido propio a la misma. De igual forma, hay que considerar a los profesionales y los familiares. Por lo que el modelo de asistencia 


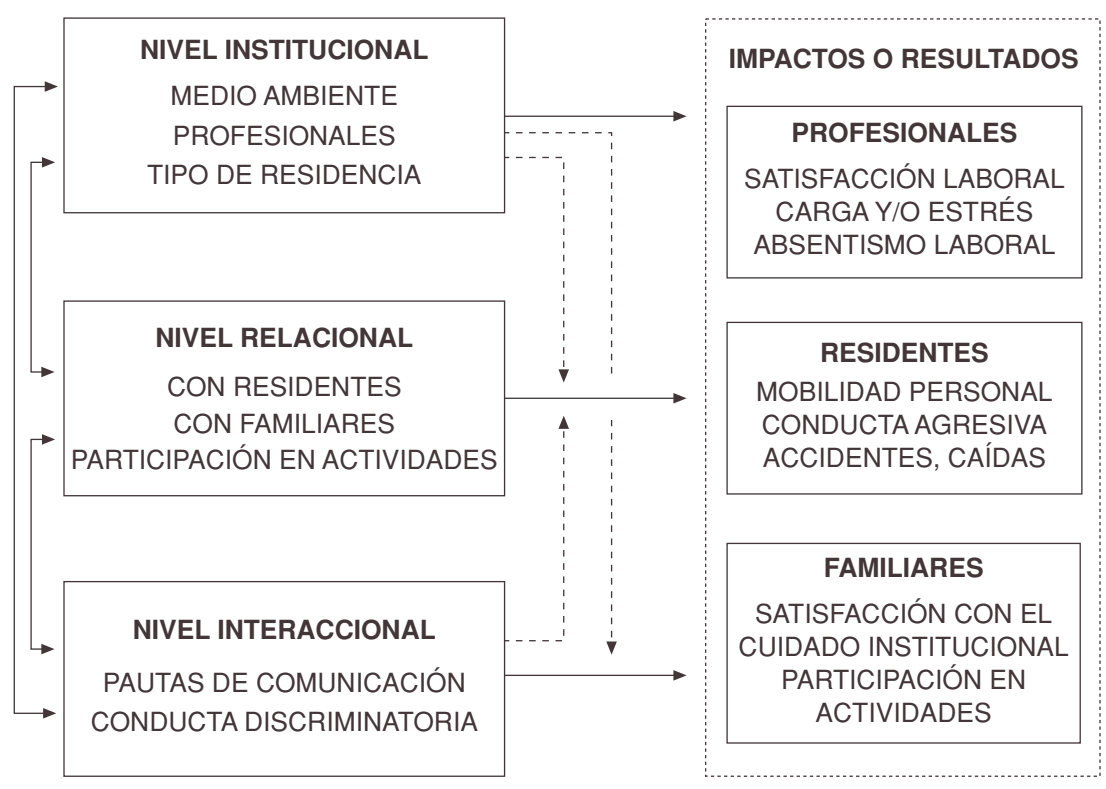

Figura 3. Modelo teórico del cuidado asistencial institucional a personas mayores y/o con discapacidad.

institucional incorpora características personales, tanto de los residentes como de los profesionales y familiares o cuidadores informales — v.g., la edad y el género, el nivel educativo, los ingresos, la estructura familiar, etc.

\section{Estudio: material y método}

El presente estudio pretende examinar el impacto de los factores mencionados previamente sobre la calidad asistencial institucional. El objetivo es el de progresar en el conocimiento de la influencia relativa de factores correspondientes, tanto al nivel institucional como al relacional e interaccional. La hipótesis central considera que los aspectos de cada uno de los niveles mencionados, aquéllos que más se identifiquen con los rasgos negativos de la «institución total» estarán asociados con resultados negativos en la asistencia a residentes, reflejándose tanto en la situación de éstos como en el grado de satisfacción de familiares y profesionales. Por ejemplo: cuando se da una mayor relación o comunicación del residente con el mundo exterior, una mejor comunicación con los profesionales o una recreación de la atmósfera familiar en el centro, se obtendrá una percepción más positiva sobre la calidad de la asistencia institucional. 


\section{a) Muestra}

Los datos utilizados para el presente estudio sobre la calidad asistencial en las residencias, forman parte de la base de datos del estudio longitudinal sobre la valoración del cuidado asistencial en las unidades especiales de atención a enfermos de alzheimer (SCU), financiado por el Instituto Nacional de la Vejez (NIA) de los Estados Unidos (Propuesta de Investigación: \#5 U01 AG10318-03), y realizado por el Centro de Gerontología de la Universidad de Kansas, Lawrence, KS (EE.UU.). El estudio incluye información sobre características de las residencias, los residentes y sus familiares, así como de los profesionales de la asistencia institucional.

La muestra contempla datos sobre cien residencias de tamaño medio — con al menos cincuenta camas - en los estados de Washington, Michigan y Carolina del Norte. La información sobre las características del centro con respecto al medio físico, al ambiente y a la composición del personal trabajador se obtuvo a través de entrevistas personales con los administradores de las residencias. Los datos sobre los residentes $(\mathrm{N}=365)$ corresponden a valoraciones realizadas por el personal de las residencias como parte del protocolo del Conjunto Mínimo de Datos (MDS), que se administra a cada residente de forma habitual (Morris y otros, 1990). Finalmente, la información de los familiares $(\mathrm{N}=352)$ proviene de una muestra no probabilística a través de un cuestionario enviado por correo. La muestra final $(\mathrm{N}=286)$ se obtuvo combinando los datos iniciales, recogidos durante 1994-95, sobre características de los centros residenciales, los residentes y familiares. La muestra incluye únicamente casos en los que existe información de los tres tipos, información de un familiar que tiene un residente en una determinada residencia. La unidad de análisis es el residente, al que se agregan los datos de su familiar. Los datos de la residencia se asignan por igual a todos los que residen en el mismo centro.

\section{b) Variables dependientes: calidad del cuidado asistencial institucional}

La calidad del centro, de la asistencia institucional, se operacionaliza con variables que corresponden al impacto sobre los residentes, familiares y profesionales:

- Satisfacción familiar: el índice sobre la percepción de familiares, de la calidad asistencial, se basa en sus respuestas sobre satisfacción ante diversos aspectos de la asistencia institucional —-según un inventario de dieciocho indicadores con escala de cuatro puntos de medida-. De las cuatro dimensiones que fueron reconocidas por el Análisis Factorial (Componentes Principales con Rotación Varimax), el índice de satisfacción general o global de los familiares se compone de ocho indicadores. Éstos hacen referencia a la preferencia de la residencia, la labor de los profesionales, la respuesta rápida a las necesidades de los residentes, la posible recomendación del centro a otras personas, la mejorabilidad de la asistencia otorgada por los profesionales, la calidad del medio ambiente o del entorno 
del centro, su configuración y las tareas de la asistencia institucional (Cronbach's alpha: $r=0,87$ ).

- Valoración general de la residencia: fue realizada, tanto por los familiares como por los administradores, utilizando un instrumento de ocho indicadores con escala de diez puntos — el valor de 1 indica una valoración negativa y diez positiva. El Análisis Factorial extrajo un solo factor para los ocho indicadores. Las variables hacen referencia a aspectos sobre la interacción de los profesionales con los residentes, el grado de participación de éstos en las actividades del centro, la opinión sobre el entorno físico, el aseo y mantenimiento del centro, su ambiente, configuración, diseño y grado de comodidad. El nivel de consistencia o fiabilidad (Cronbach's Alpha) para la muestra de los familiares fue de $r=0,87(\mathrm{~N}=352)$, y algo más elevada para la de los administradores: $r=0,91(\mathrm{~N}=100)$.

- Retención de los profesionales: se operacionaliza como un índice de precariedad media del profesional auxiliar por centro y representa el porcentaje total del número de profesionales que no regresa a su puesto de trabajo sobre aquéllos que lo hacen. El índice de retención de los trabajadores se considera una medida del tipo y nivel de calidad asistencial de cada centro. La presunción es que el nivel de calidad del centro influye sobre la moral y conducta de los profesionales, anticipándose que a mayor calidad institucional corresponda mayor retención de los profesionales.

- Participación del residente en actividades: el tiempo que los residentes dedican a realizar actividades se considera un dato del nivel de calidad asistencial del centro. Del protocolo del Conjunto Mínimo de Datos (MDS) se toma el indicador de tiempo medio que el residente dedica a la realización de actividades. La escala de respuesta es: nunca, menos de $1 / 3$ del tiempo, entre $1 / 3$ y $2 / 3$ del tiempo, y más de $2 / 3$ del tiempo.

\section{c) Variables independientes: factores determinantes de la calidad}

Las variables independientes utilizadas en el estudio corresponden a los tres niveles:

- El institucional, que incluye las siguientes características del centro: 1) el carácter público o privado —ánimo de lucro—; 2) la limpieza general del entorno físico, valorado con un índice de diez indicadores sobre la limpieza general, ruidos, olores, iluminación, limpieza y mantenimiento general $(r .=0,88)$; 3$)$ la configuración familiar, que se compone de cinco indicadores sobre el ambiente residencial, preguntando por la accesibilidad del residente a la cocina, la existencia de obstáculos en pasillos, comodidad de las habitaciones, y la posibilidad de estimulación táctil y visual del residente $(r .=0,60) ; 4)$ el porcentaje total de profesionales, que incluye el conjunto de trabajadores promedio en el centro por cama a cualquier hora de la jornada; 5) el nivel de entrenamiento de los profesionales para combatir el estrés laboral, medido con un índice total de horas de formación imparti- 
das por el centro, y 6) la retención de los profesionales o índice de precariedad media del profesional auxiliar por centro, que, además de ser utilizado como un resultado de la calidad asistencial del centro, se considera igualmente un factor predictivo del nivel de satisfacción de los familiares y de la valoración de los administradores sobre la asistencia institucional.

- El relacional, que está representado por: 1) el número de visitas de los familiares; 2) la participación de los familiares en la asistencia informal a residentes, medida con ocho indicadores en escala de cinco puntos (MDS). Los resultados del Análisis Factorial (Componentes Principales y Rotación Varimax), indican la existencia de dos factores: a) la participación global de los familiares en actividades de formación, con los residentes y con los profesionales $(r .=0,77)$; $\mathrm{y} b$ ) el cuidado directo informal a su residente en tareas personales como la ayuda en las comidas, vestirse, bañarse y el aseo personal $(r .=0,76) ; 3)$ el índice de interacción de los residentes, construido en base a seis indicadores (MDS), sobre su facilidad y deseo para tratar a los otros, participar e iniciar actividades, proponer sus propios objetivos $(r=0,62)$, y 4) la participación del residente en actividades del centro, presentada anteriormente como una de las variables dependientes, y que se utiliza igualmente como factor predictivo de la calidad asistencial institucional.

- El interaccional, representado por el nivel de comunicación y el tipo de relaciones conflictivas de los residentes: 1) habilidades de comunicación: medido con un índice compuesto por dos indicadores del protocolo del Conjunto Mínimo de Datos (MDS), con una escala de respuesta de cuatro puntos - nunca se hace entender, a veces se hace entender, con dificultad y completamente entendido o comprendido-, sobre la habilidad de expresarse y de hacerse entender por los otros $(r .=88)$, y 2$)$ relaciones conflictivas: que se forma con cuatro indicadores (MDS) sobre las relaciones de conflicto, abierto o encubierto, con los otros residentes, con los profesionales del centro, con los compañeros de habitación, con los familiares y amigos $(r .=0,51)$.

\section{d) Variables de control: factores sociodemográficos}

Los factores de control incluyen características, tanto de los residentes como de los familiares, respecto del género, la edad, el estado marital y la relación de parentesco. También se consideran el nivel educativo de los familiares, así como los niveles de necesidad funcional, movilidad, atención personal y deterioro cognitivo de los residentes.

Los instrumentos utilizados para la medición de las variables de necesidad de los residentes se toman del protocolo del Conjunto Mínimo de Datos (MDS), e incluyen: 1) movilidad, formado por cuatro indicadores de actividades instrumentales de la vida diaria sobre asistencia para moverse, ordenar la cama, cambiar de posición (de la cama a la silla, etc.), desplazarse, y para comer y beber $(r .=0,89) ; 2)$ cuidado personal, que es un índice de cinco indicadores de actividades básicas de la vida diaria sobre la necesidad de ayuda en el uso del baño, vestirse, bañarse, incontinencia urinaria y aseo personal $(r .=0,88)$. 
La escala de respuesta de los indicadores de ambos índices de necesidad es de cinco puntos: independiente, necesita supervisión, asistencia limitada, moderada y completa.

\section{Resultados}

Las características de edad, género y estado civil de los participantes en la muestra, tanto de los residentes como de los familiares, se presentan en la tabla 1. La muestra final incluye 286 residentes, con una edad promedio de 82,6 años $(\mathrm{SD}=8,7)$, contando más de la mitad de los mismos por encima de 83 años, de los que un 19,6\% pertenece al grupo de los más adultos — 90 años y más. Los residentes son mayoritariamente mujeres $(68,2 \%)$ y tan sólo el 35\% se encuentra casado/a. Los familiares presentan una edad promedio de 62,1 años, siendo en sus dos terceras partes mujeres $(61,2 \%)$, y en su inmensa mayoría, casadas $(80,4 \%)$. Más de la mitad de familiares cuidadores son las hijas/os $(56,3 \%)$ de los residentes, con tan sólo un 26,6\% de esposas/os, y en menor medida $(17,1 \%)$ otros familiares o amigos. Los familiares llevan realizando tareas como cuidadores informales por un período medio de uno a dos años.

La determinación de la influencia de los factores a nivel institucional, relacional e interaccional, se realiza a través del Análisis de la Regresión Múltiple, con el análisis de la regresión de los factores predictivos sobre cada uno de los impactos o resultados de la asistencia institucional. La estimación del modelo predictivo, a través del análisis de la regresión, se realiza en una sola etapa, de

Tabla 1. Características de la muestra $(\mathrm{N}=286)^{*}$

\begin{tabular}{llrrrr}
\hline \multirow{2}{*}{ Características } & & \multicolumn{2}{c}{ Familiares } & \multicolumn{2}{c}{ Residentes } \\
\cline { 3 - 6 } Edad & & $\mathrm{N}$ & $\%$ & $\mathrm{~N}$ & $\%$ \\
& $<=50$ & 50 & 17,5 & 1 & 0,3 \\
& $50-59$ & 76 & 26,6 & 3 & 1,0 \\
& $60-69$ & 68 & 23,8 & 16 & 5,6 \\
& $70-79$ & 57 & 19,9 & 67 & 23,4 \\
& $80-89$ & 24 & 8,4 & 142 & 49,7 \\
\multirow{2}{*}{ Género } & $=>90$ & 4 & 1,4 & 56 & 19,6 \\
\multirow{2}{*}{ Estado civil } & Hombre & 109 & 38,1 & 86 & 30,1 \\
& Mujer & 175 & 61,2 & 195 & 68,2 \\
& Casado/casada & 230 & 80,4 & - & - \\
& Soltero/soltera & 10 & 3,5 & 100 & 35,0 \\
Relación familiary & Viudo/viuda & 20 & 7,0 & 13 & 4,5 \\
& Otro & 26 & 9,1 & 156 & 54,5 \\
& Esposo/esposa & 76 & 26,6 & - & - \\
& Hijo/hija & 161 & 56,3 & - & - \\
& Otro & 49 & 17,1 & - & - \\
\hline
\end{tabular}

* La muestra se compone de igual número de familiares $(\mathrm{N}=286)$ que de residentes $(\mathrm{N}=286)$. 
forma simultánea para todas las variables. Los modelos estimados fueron cinco: en la tabla 2 se presentan los resultados para cada uno de ellos.

La calidad de la asistencia institucional según el grado de satisfacción de los familiares, está determinada por factores tanto del nivel relacional como

Tabla 2. Análisis de regresión múltiple: factores que determinan la calidad de la asistencia institucional (1).

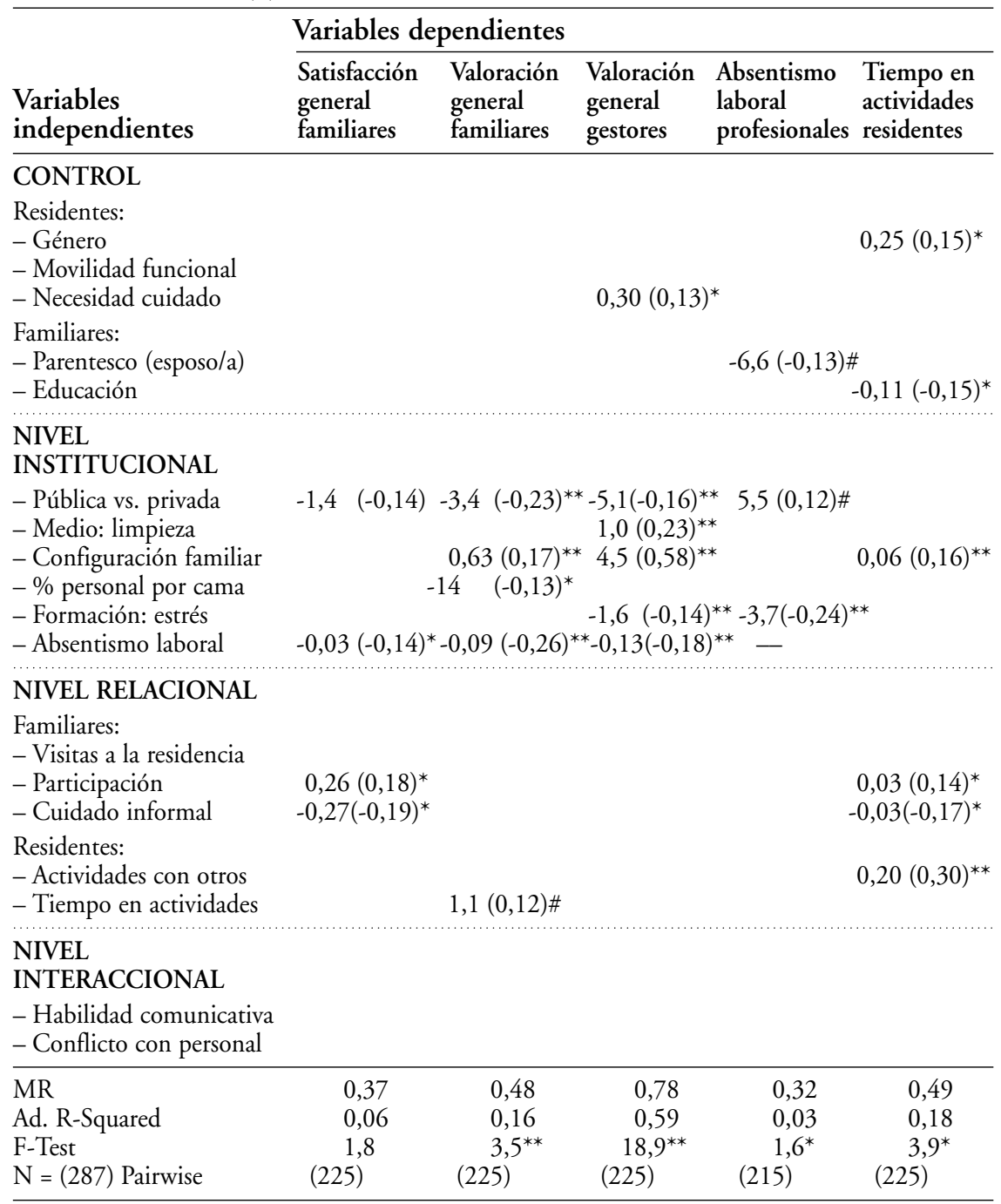

${ }^{* *} \mathrm{p}<0,01 ;{ }^{*} \mathrm{p}<0,05 ; \quad \# \mathrm{p}<0,10$

(1) Unstandardized Regression Cooefficients (Betas: Standardized Coefficients). 
institucional. El primer modelo explicativo, que considera los factores que predicen la calidad asistencial según es percibida por los familiares, tan sólo alcanza a explicar el 6\% de la varianza total. Los aspectos predictivos más importantes corresponden a los dos factores del nivel relacional: el primero es la participación de los familiares como cuidadores informales, realizando tareas de cuidado personal o de atención directa a residentes, en el sentido de que los familiares expresan un nivel menor de satisfacción general con la asistencia institucional cuando su participación como cuidadores informales es mas alta; y el segundo es la participación general de los familiares en las actividades del centro, indicando que dicha participación está asociada, en menor medida pero de forma igualmente significativa, a un nivel más alto de satisfacción con la asistencia institucional.

Junto a estos factores, existen otros dos aspectos del nivel institucional que son significativos: el primero es el carácter del centro, siendo el centro privado o con ánimo de lucro el asociado con un nivel más bajo de satisfacción familiar, es decir, los que se consideran con menor calidad asistencial; y el segundo se refiere a la capacidad de retención de los profesionales de la asistencia institucional. Los familiares expresan un nivel más alto de satisfacción cuando perciben una estabilidad de los profesionales, de aquéllos que realizan el cuidado continuo de los residentes.

Los modelos predictivos sobre la valoración, tanto por parte de los familiares como de los administradores sobre la condición general del centro y de la asistencia institucional, aportan mayores porcentajes de explicación $-16 \%$ y $59 \%$ respectivamente. En ambos modelos son los aspectos del nivel institucional los que prioritariamente determinan la valoración. En particular, los familiares valoran la calidad asistencial en centros donde se da una mayor retención de los profesionales sociosanitarios, son públicos, existe una configuración y un ambiente familiar para los residentes, y se da un porcentaje no muy elevado de personal profesional por cama o residente. Los familiares también perciben como positivo la participación y una mayor dedicación del tiempo de los residentes en las actividades del centro.

Por otra parte, los administradores reconocen las características medio ambientales respecto de la configuración, la limpieza y el mantenimiento como los aspectos más importantes en la valoración positiva del centro. La valoración general resultó asociada igualmente con el carácter del centro, siendo los públicos, o centros sín ánimo de lucro, los que mejor valoración reciben; con un menor índice de absentismo, esto es, los centros con mejor retención de los profesionales son los que reciben una valoración más positiva; y en general con centros donde se dedica menos tiempo a la formación o entrenamiento de los profesionales sobre el estrés laboral. Dado que los datos sobre la valoración se realizó a nivel de centro, el modelo predictivo también se examinó utilizando la muestra de centros $(\mathrm{N}=100)$, incluyendo sólo los factores predictivos del nivel institucional. Los resultados del Análisis de la Regresión Múltiple fueron similares, representando una réplica de los resultados obtenidos con la muestra de residentes y familiares $(\mathrm{N}=286)$. En definitiva, son los 
factores del nivel institucional los que fundamentalmente determinan la valoración sobre la calidad de la asistencia según los administradores en estas instituciones.

El modelo predictivo con respecto a la calidad de la asistencia institucional según el grado de retención o absentismo laboral de los profesionales alcanza el nivel predictivo más bajo (3\%). La formación y el entrenamiento de los profesionales sobre el estrés en las tareas asistenciales que realizan en los centros es el factor más relevante para lograr la retención de los trabajadores. Igualmente, éstos tienen, en los centros residenciales públicos, un índice superior de retención en el trabajo que en los privados. Es necesario hacer notar que existen otros factores importantes que influyen en el índice de retención laboral y, por extensión en la calidad asistencial, y que no han sido tenidos en cuenta en el modelo. Por ejemplo, el nivel educativo de los trabajadores, su nivel de ingresos o sus condiciones estructurales en el trabajo - turnos, etc. - son factores potenciales que han de ser examinados junto a los utilizados en el presente análisis.

Finalmente, el modelo predictivo del impacto de la calidad institucional sobre los residentes indica que tanto factores del nivel relacional como institucional intervienen en la determinación de la cantidad de tiempo que los residentes dedican a actividades. El modelo explica en conjunto un $18 \%$ de este resultado. El índice de interacción o de actividades con otros residentes, es el que aporta más peso en la explicación del tiempo medio dedicado por el residente a actividades. Junto a este factor relacional de los residentes el cuidado informal que realizan los familiares también está asociado de forma significativa a un menor tiempo dedicado a actividades.

Por el contrario, la mayor participación de los familiares en las actividades del centro ocasiona una mayor dedicación de tiempo a actividades por parte del residente. El único aspecto institucional que se asocia positivamente con el tiempo dedicado a actividades es el de la configuración familiar del centro, indicando que los residentes dedican más tiempo a actividades cuando se da una configuración o un ambiente que se hace más propio de las características de tipo familiar. Por último, resaltar que, de entre los residentes, son las mujeres las que de forma significativa dedican mayor tiempo a participar en las actividades del centro.

\section{Discusión}

La hipótesis principal de este estudio considera que la calidad de la asistencia institucional a los mayores está determinada por factores del ámbito institucional, relacional e interaccional, y que los resultados o impactos de los mismos han de ser reconocidos entre familiares, profesionales y residentes. Igualmente, el estudio, anticipaba que los aspectos predictivos que se identifican con características propias de la residencia, en cuanto «institución total», serían críticos en la determinación de la calidad asistencial. Los resultados del presente estudio confirman sólo en parte estas expectativas. 
Los factores más importantes asociados con la calidad asistencial, en el presente estudio fueron los aspectos del nivel institucional —el carácter privado del centro, el medio ambiente, la configuración familiar, el número de profesionales por cama, su formación y entrenamiento para combatir el estrés laboral y el grado de retención laboral-, seguidos de los indicadores del nivel relacional - participación y cuidado informal de los familiares-. Sin embargo, en contra de lo que se esperaba, los factores del nivel interaccional - habilidad de comunicación y conflicto personal - no fueron significativos respecto de la calidad asistencial, o al menos no parecen tener una influencia decisiva según los resultados analizados.

El examen de los modelos predictivos de la calidad asistencial institucional parece manifestar la existencia de diversas perspectivas sobre la importancia e influencia que se atribuye a los diversos factores predictivos. La ponderación que familiares y administradores realizan de la calidad institucional se concreta en criterios diferentes: para los familiares el énfasis se centra en las variables de participación en el plan o programa de la asistencia institucional, así como en la preocupación por la retención laboral de los profesionales. Por contra, los administradores, a diferencia de familiares, residentes y profesionales, realizan la valoración de la asistencia institucional otorgando prioridad a factores tales como la configuración del medio, el mantenimiento y la limpieza del entorno. La coincidencia entre familiares y residentes, respecto de los aspectos relacionales, es un dato revelador de la diferente jerarquía de prioridades.

Los resultados sobre la satisfacción de los familiares, y sobre el tiempo medio que dedican los residentes a actividades, confirman la importancia de mantener las redes de apoyo informal. En la medida que las residencias neutralizan las tendencias negativas de la «institución total», en el sentido de evitar el aislamiento que caracteriza a dichas instituciones, se puede esperar que se avance en la calidad de la asistencia institucional. Es por ello interesante hacer notar que el nivel relacional aporta un componente muy importante, produciendo el resultado positivo que se pretende: la calidad de la vida en las residencias para ancianos. De igual modo, hay que pensar que se producirán resultados positivos similares como consecuencia de todo esfuerzo, o estrategia, que ayude a personalizar el medio o entorno del residente. Esta personalización evitaría la presión sobre las tendencias a la rutina, la excesiva reglamentación y pérdida del control personal, que son aspectos negativos propios de toda «institución total».

Las razones por las que los factores del nivel interaccional no fueron estadísticamente significativos, en la determinación de la calidad institucional, pueden ser muy variadas. Sin duda, uno de los argumentos importantes reside en el moderado nivel psicométrico de las variables del nivel interactivo que han sido utilizadas en el presente análisis. Por ello, sería necesario, para lograr la ponderación de estos factores, el desarrollo de instrumentos válidos y fiables, que adecuadamente operacionalicen, midan, los conceptos sobre los estilos de comunicación entre residentes y profesionales sociosanitarios, así como otros factores de este nivel. 
Los modelos teóricos de la calidad asistencial institucional, a pesar de contar con varios factores predictivos, sólo alcanzaron un nivel moderado de explicación de la varianza de algunas variables dependientes. Este resultado parece indicar que existen aspectos importantes que no han sido incluidos en el modelo teórico inicial. El impacto de los factores institucionales, relacionales e interaccionales sobre el grado de satisfacción de los residentes, familiares y profesionales podrían estar mediatizados por factores como el de las creencias, valores y expectativas que los familiares tienen sobre la asistencia institucional. Las expectativas son variables a tener en cuenta en el grado de satisfacción de los familiares con respecto al cuidado institucional que reciben los residentes. Por ejemplo, el estudio de Biedenharn y Normoyle (1991) señaló que la actitud de los familiares ante la posibilidad de ingresar a un familiar en una residencia tiene que ver con las expectativas sobre el entorno físico institucional, sobre el papel asignado por los administradores del centro a los familiares en la provisión del cuidado, así como con la percepción que éstos tienen sobre la calidad asistencial institucional.

Finalmente, es importante señalar que los efectos de los factores que determinan la asistencia institucional presentados anteriormente se formulan como efectos directos. Sin embargo, parece más lógico y adecuado con la realidad el anticipar un modelo más complejo en el que se incluyan las interacciones entre varios de los factores enumerados. Dichas interacciones - ver en la figura 1 las posibles relaciones de interacción indicadas con las flechas punteadas-, nos darían una visión mucho más conforme con los procesos sociales y, al mismo tiempo, nos ayudarían a reconocer las condiciones específicas que posibilitan la calidad de la asistencia institucional a los mayores. Por ejemplo, las variables del nivel institucional, como son las del medio físico o del tipo de centro - público o privado- - pueden contribuir en la obtención de resultados positivos en la calidad asistencial, pero siempre y cuando esta relación sea posible bajo la condición de otro factor como, pongamos por caso, el de una intensa participación de los cuidadores informales en la toma de decisiones, en la programación e implementación de las actividades del centro.

En definitiva, sólo cuando somos capaces de incorporar los diversos niveles del análisis social a nuestros modelos teóricos, y de realizar la sincronización de ambos niveles, podemos llegar a comprender mejor los procesos sociales. La calidad de la asistencia institucional no puede, pues, ignorar ni al individuo ni a su mundo social, siendo necesario para su consecución prestar atención a cada residente, tanto desde el prisma de su biografía como de su entorno e historia.

\section{Bibliografía}

Attias-Donfut, C. (1992). «Dependencia de las personas mayores y ayuda intergeneracional». Papers, 40: 13-33.

BARENYS, M. Pia (1993). «Un marco teórico para el estudio de las instituciones de ancianos». Revista Española de Investigaciones Sociológicas, 64: 155-172. 
BAzo, M.T. (1991). «Institucionalización de personas ancianas». Revista Española de Investigaciones Sociológicas, 53: 149-164.

Biedenharn, P.J.; Normoyle, J.B. (1991). «Elderly Community Residents' Reactions to the Nursing Home: An Analysis of Nursing Home-Related Beliefs». The Gerontologist, 31(1): 107-115.

Caporael, L. (1981). "The Paralanguage of Caregiving: Baby Talk to the Institutionalized Aged». Journal of Personality and Social Psychology, 40: 876-884.

Chappell, N.L.; NovaK, M. (1992). "The Role of Support in Alleviating Stress among Nursing Assistants». The Gerontologist, 32(3): 251-359.

Chenoweth, B.; Spencer, B. (1986). "Dementia: The Experience of Family Caregivers". The Gerontologist, 26: 269-272.

Centro de Investigaciones SOCIOlógiCas (1990). «Situación social de los viejos». Estudios y Encuestas, 21. Madrid: CIS.

Coupland, N.; Coupland, J.; Giles, H. (1991). Language, Society and the Elderly. Cambridge, MA: Blackwell.

DunCan, M.T.; Morgan, D.L. (1994). "Sharing the Caring: Family Caregivers' Views of Their Relationships with Nursing Home Staff». The Gerontologist, 34(2): 235- 244

Estes, C.L. (1979). The Aging Enterprise. San Francisco: Jossy-Bass.

Estes, C.L.; Swan, J.H.; Associates (1993). The Long Term Care Crisis. Elders Trapped in the No-Care Zone. Newbury Park (CA): Sage.

Folger, J.P.; Poole, M.S.; Stutman, R. (1993). Working through Conflict: A Communication Perspective. Glenview, IL: Scott, Foresman, and Co.

Foner, N. (1994). «Nursing Home Aides: Saints or Monsters?». The Gerontologist, $34(2): 245-250$.

Fundación Caja Madrid (1996). Envejecer en España. Manual estadístico sobre el envejecimiento de la población. Madrid: Fundación Caja Madrid.

George, L.K.; MAdDoX, G.L. (1989). «Social and Behavioral Aspects of Institucional Care». En Ory, M.; Bond, K. (eds.). Aging and Health Care. Londres: Routledge. Goffman, E. (1961). Asylums. Nueva York: Doubleday.

Hocker, J.L.; Wilmot, W.W. (1995). Interpersonal Conflict (4th ed.). Dubuque, IA: William C. Brown.

INSERSO (1995). Las personas mayores en España. Perfiles. Reciprocidad familiar. Madrid: Instituto Nacional de Servicios Sociales.

Itschaki, N. (1994). "Occupational Stresses and Burnout Among Hospital Social Workers». Unpublished Dissertation, Columbia University, Department of Social Work, Febrero, 1994.

Kane, R.L.; Kane, R.A. (1978). "Care of the Aged: Old Problems in Need of New Solutions». Science, 200(26): 913-919.

- (1990). «Health Care for Older People: Organizational and Policy Issues». En Binstock, R.H.; George, L.K. (eds.). Handbook of Aging and Social Science. Nueva York: Academic Press.

Kahana, E. (1982). «A Congruence Model of Person-Environment Interactions». En LawTon, M.P. y otros (eds.). Aging and the Environment: Theoretical Approaches. Nueva York: Springer.

- (1987). «Institutionalization». En Maddox, G.L. (ed.). Encyclopedia of Aging. Nueva York: Springer. 
KaHANA, E.; KaHANA, B. (1983). «Environmental Continuity, Discontinuity, Futurity and Adaptation of the Aged». En Rowles, G.; OHTA, R. (eds.). Aging and Milieu: Environmental Perspectives on Growing Old. Nueva York: Academic Press.

KAHANA, E.; KAHANA, B.; YOUNG, R. (1985). «Social Factors in Institutional Living». En Quadagno. J. (ed.). Social Bonds in Later Life: Aging and Interdependence. Beberly Hills: Sage.

KemPER, S. (1994). «Elderspeak: Speech Accommodations to Older Adults». Aging and Cognition, 1: 1-10.

LAWTON, M.P. (1980). Environment and Aging. Monterey (CA): Brooks/Cole.

LaWTON, M.P.; Moos, M.; Fulcomer, M.; Kleban, M.H. (1982). «A Reserach and Service Oriented Multilevel Assessment Instrument». Journal of Gerontology, 37(1): 91- 99.

Lieberman, M.A.; Tobin, S.S. (1983). The Experience of Old Age: Stress, Coping and Survival. Nueva York: Basic Books.

LITWAK, E. (1985). Helping the Elderly: The Complementary Roles of Informal Networks and Formal Systems. Nueva York: Guilford.

Litwak, E.; Messeri, P.;. Silverstein, M. (1990). «The Role of Formal and Informal Groups in Providing Help to Older People». En Under, D.; Sussman, M. (eds.). Families in Community Settings: Interdisciplinary Perspectives. Nueva York: Haworth.

LYNCH, S.A. (1994). "Job Satisfaction of Home Health Nurses». Home Health Nurse, 12(5): 21-28.

MaAs, M.L.; BucKwalter, K.C.; Kelley, L.S. (1991). «Family Members' Perceptions of Care of Institutionalized Patients with Alzheimer's Disease». Applied Nursing Research, 4(3): 135-140.

MASLOW, K. (1994). "Current Knowledge about Special Care Units: Findings of a Study by the U.S. Office of Technology Assessment». Alzheimer Disease and Associated Disorders, 8(1): 14-40.

Montgomery, R.J.V. (1982). "The Impact of Institutional Care Policies on Family Integration». The Gerontologist, 27(1): 54-58.

Montgomery, R.J.V.; Montoro, J. (1995). «Family Members as the "Other Clients" in Nursing Home Settings". Paper delivered at the Gerontological Society of America (GSA). Los Angeles.

Montoro, J. (1995). «The Role of Family Members in the Special Care Unit Context». Paper delivered at the Mid-America Congress on Aging (MACA). Kansas City.

Morris, J.N.; HaWes, C.; Fries, B.E.; Phillips, C.D.; Mor, V.; KatZ, S.; Murphy, K.; Drugovich, M.L.; FrIedlob, A.S. (1990). «Designing the National Resident Assessment Instrument for Nursing Homes». The Gerontologist, 30: 293-307.

Moos, R.H.; Lemke, S. (1984). Multiphasic Environmental Assessment Procedure: Mannual. Palo Alto(CA): Veterans Administration.

Moos, R.H.; Gauvain, M.; LemKe, S.; MaX, W.; Mehren, B. (1979). «Assessing the Social Environments of Sheltered Care Settings». The Gerontologist, 19(1): 74- 82.

Noelker, L.; Harel, Z. (1978). "Predictors of Well-being and Survival Among Institutionalized Aged». The Gerontologist, 18(6): 562-567.

NussBaum, J.F. (1991). «Communication, Language and the Institutionalized Elderly». Aging and Society, 11: 149-165.

PARRY, J.K.; SMITH, M.J. (1987). «A Study of Social Workers' Job Satisfaction as Based on an Optimal Model of Care for the Terminally Ill». Journal of Social Service Research, 11(1): 39-58. 
Pillemer, K.; Hudson, B. (1993). «A Model Abuse Prevention Program for Nursing Assistants». The Gerontologist, 33(1): 128-131.

Pratt, C.; Schmall, V.; Wright, S.; Hare, J. (1987). «The Forgotten Client: Family Caregivers to Institutionalized Dementia Patients». En BrubaKer, T. (ed.). Aging, Health and Family: Long Term Care. Beverly Hills, CA: Sage.

PraY, J.E. (1993). "Fostering Effective Interaction Between Nursing Home Staff and Residents Experiencing Alzheimer's Related Dementia». Doctoral Dissertation. University of Kansas.

Putnam, L.L.; WiLson, C.E. (1982). «Communicative Strategies in Organizational Conflicts: Reliability and Validity of a Measurement Scale». En Burgoon, M. (ed.). Communication Yearbook, 6. New Brunswick, NJ: Transaction.

Quadagno, J. (1982). Aging in Early Industrial Society: Work, Family and Social Policy in Nineteenth Century England. Nueva York: Academic Press.

Rubin, A.; Shuttlesworth, G. (1983). «Engaging Families as Support Resources in Nursing Home Care: Ambiguity in the subdivision of Trasks». The Gerontologist, 23: 632-636.

Small, Jeff; Montoro Rodríguez, J.; Kemper, S. (1996). «Discourse Styles of Conflict Resolution in a Nursing Home Setting." International Conference on Communication, Aging and Health (ICCAH). Lawerence, KS., mayo.

Tobin, S.S. (1989). "The Effects of Institutionalization". En. MArkides, K.S.; Cooper, C. (eds.). Aging, Stress and Health. Nueva York: Wiley.

Walker, A. (1981). "Towards a Political Economy of Old Age». Ageing and Society, 1(1): 73-94. 